\title{
Annabelle KLEIN, dir., Nos jeunes à l'ère numérique
}

Louvain-la-Neuve, Academia/Éd. L'Harmattan, coll. Pixel, 2016, 206 pages

Florence Michet

\section{OpenEdition}

\section{Journals}

Édition électronique

URL : http://journals.openedition.org/questionsdecommunication/11726

DOI : 10.4000/questionsdecommunication. 11726

ISSN : 2259-8901

\section{Éditeur}

Presses universitaires de Lorraine

\section{Édition imprimée}

Date de publication : 31 décembre 2017

Pagination : 470-473

ISBN : 9782814305076

ISSN : 1633-5961

\section{Référence électronique}

Florence Michet, «Annabelle kLEIN, dir., Nos jeunes à l'ère numérique », Questions de communication [En ligne], 32 | 2017, mis en ligne le 31 décembre 2017, consulté le 05 janvier 2021. URL : http:// journals.openedition.org/questionsdecommunication/11726; DOI : https://doi.org/10.4000/ questionsdecommunication. 11726 
Cependant, il ne faut pas croire que l'ouvrage, bien qu'il le soit souvent, soit toujours aussi drôle. Et, précisément, la mise en spectacle de certains événements, comme la commémoration du débarquement le 6 juin 2014 (pp. 234-236), où une chorégraphie symbolique parait choquante à l'auteur : « J'avoue qu'à ce moment j'ai eu un peu honte... N'y avait-il rien d'autre à proposer à nos visiteurs que cette chorégraphie approximative qui traitait finalement avec légèreté et lourdeur des moments aussi forts de notre histoire au risque de paraitre confondre une émission de télévision et un hommage rendu à ceux qui se sont sacrifiés pour nous 》». Même réaction face au manque de respect de la dignité humaine comme ce fut le cas pour l'assassinat, filmé en direct, d'Ahmed Merabet, un policier abattu par les terroristes boulevard Richard Lenoir, lors de l'attaque de Charlie-Hebdo en février 2013 (pp. 291-293).

Certains hommes politiques bénéficient d'un intérêt particulier lié à la permanence de leur présence. Ainsi, dans l'index des noms, Jacques Chirac est-il encore cité 12 fois quand Nicolas Sarkozy l'est 45 fois et François Hollande 40, entre 2012, alors que le premier vient d'être battu à l'élection présidentielle, et 2016, avant-dernière année du quinquennat du second. On voit aussi apparaître 12 fois Marine Le Pen qui assure la permanence du Front national, 7 fois Jean-Luc Mélenchon, futur candidat déclaré à l'élection de 2017. Ces chiffres, dans leur sécheresse, témoignent de la puissance politique que représentent les médias grand public, ici la télévision, et permettent de s'interroger sur leur rôle. Si l'on ne peut pas en tirer de conclusions sur les forces politiques réelles, on peut du moins sentir comment se construit l'image des uns et des autres, et les conséquences qu'il conviendrait d'en tirer. Fin 2016, Nicolas Sarkozy sera éliminé aux primaires de la droite, tandis que François Hollande jugera plus prudent de ne pas se représenter. Cela ne veut pas dire que François Jost prévoit l'avenir, mais il nous permet de mieux comprendre le rôle des médias dans les mécanismes de la prise de pouvoir.

Ces évaluations chiffrées sont loin de faire le tour d'un ouvrage qui insiste aussi sur les effets des émissions diffusées. Ainsi les modalités et la signification plus ou moins troublante du succès des élucubrations d'un «Éric Zemmour, le showman du populisme » sontelles analysées (p. 265). Ou bien les effets pervers de la téléréalité à propos du comportement de certains candidats qui dérapent dans la vie réelle (« L'agression de Nabila : la téléréalité rend-elle fou ? », p. 268). D'autres textes s'inquiètent : ainsi dans « Le mensonge est-il un péché médiatique » (p. 3|4), l'auteur interroget-il la «moralisation du mensonge » où il voit un signe « de l'américanisation de nos médias » (p. 316). II a aussi ses têtes de turc, si j'ose dire : ainsi Nicolas Sarkozy est-il particulièrement servi en juin-juillet 2015 avec « Sarkozy dans son show » (p. 326), « Sarkozy et la stratégie du coucou » (p. 329), « Sarkozy dans Match » (p. 331) après avoir déjà eu droit à 3 textes en rafale (pp. 256-265) avec « Le retour de Sarkozy (épisode 2) 》, « Sarkozy et I'histoire » et « Sarkozy et ses amis au stade : le retour du bling-bling »! Pas d'hypocrite neutralité, mais des coups de boutoir ciblés; on le voit, François Jost ne prétend pas à « l'impartialité du chercheur $»$, mais se sert de ce travail pour exprimer ses indignations et formuler ce que pourrait être une « éthique des médias » sans hypocrisie.

Souvent réjouissant pour les téléspectateurs(trices) qui préfèrent l'ironie à l'admonestation, l'ouvrage se termine sur un « éloge de la sémiologie », hommage rendu à Umberto Eco. Ce dernier est présenté comme un « être gai, pétillant d'humour, toujours prêt à raconter une histoire drôle » (p. 374). Tel qu'on peut l'imaginer grâce au roman de Laurent Binet, La septième fonction du langage (Paris, Librairie générale française, 2015), où il pérore dans un café sur la « fonction magique » du langage « qui a toujours été l'enjeu politique fondamental » (p. 222). Par la suite, François Jost rend hommage à celui qui a su être à la fois « le romancier, le professeur, le sémiologue » et réconcilier « le penseur et l'artiste » (p. 376) en conciliant lui-même la morale et la verve à travers « des chiffres et des lettres» pour nous offrir une réflexion aussi plaisante que féroce.

Michèle Lagny

Ircav, université Sorbonne Nouvelle - Paris 3, F-75231 michelelagny@sfr.fr

\section{Annabelle KLeIN, dir., Nos jeunes à l'ère numérique}

Louvain-la-Neuve, Academia/Éd. L'Harmattan, coll. Pixel, 2016, 206 pages

À l'heure où beaucoup de questions se posent sur les rapports des jeunes et du numérique, cet ouvrage essaie de donner des clés aux éducateurs pour les accompagner. Coordonné par Annabelle Klein, spécialiste des questions d'identité numérique, il propose un regard centré sur les « pratiques jeunes » et la place occupée par les écrans dans leur environnement. Sa composition - plusieurs contributions d'auteurs issus de disciplines différentes: philosophie, psychologie, sciences de l'information, communication, psychothérapie - le rend dynamique et complet. De plus, il promet de s'adresser à un public non averti, enseignants et parents. Nous attendons 
donc un langage accessible « dans un souci de vulgarisation scientifique et d'utilité sociale » (p. 16). La préface de Serge Tisseron (pp. I | - |4), psychiatre et spécialiste des relations aux objets technologiques avec écran, nous éclaire sur les intentions précises et repère trois axes menant la réflexion : accompagner; repenser les écrans selon leurs finalités ; valoriser les pratiques de création. « Cet ouvrage nous invite à faire confiance à nos enfants » (p. 14) tout en restant vigilants. La coordinatrice de l'ouvrage, en introduction (pp. 15-20), insiste sur ce croisement de regards disciplinaires essentiel pour une perspective globale d'éducation aux médias numériques. Cet essai repose sur des dualités engendrées par le numérique : diabolisation/idéalisation, intimité/extimité, usages problématiques/normaux, technophobes/technophiles, discours pessimistes/ optimistes : il ne s'agit pas de choisir l'un ou l'autre des camps mais de composer avec ces dualités. Ce livre se décline en six chapitres, chacun proposant une contribution rédigée par un ou deux auteurs avec sa propre bibliographie. II fait le tour de la vie numérique d'un « jeune », de l'enfance à l'adolescence, du contexte d'accélération des changements sociotechniques aux usages excessifs en passant par la construction d'une identité numérique, l'évolution psychoaffective, les aspects intimes et même sexuels, les réseaux sociaux et le lien social, les comportements compulsifs face aux jeux vidéo. Au fil de la lecture, les divers aspects de la vie d'un jeune se développent et quelques conseils sont donnés pour les aider. Cet écrit est présenté comme un document d'accompagnement.

Le chapitre I (pp. 21-36) rappelle les changements visibles dans tous les domaines de la vie humaine engendrés par internet et les nouveaux outils, établissant ainsi une liste des notions à envisager pour mieux accompagner les jeunes aujourd'hui. Les discours actuels font état tantôt d'un recul tantôt d'un progrès opposant « technophiles et technophobes » (p. 2l). II convient de ne pas prendre position mais plutôt de s'intéresser à quelques notions importantes concernant le développement de l'enfant : la notion de « digital natives » de Marc Prensky toujours bien ancrée dans les esprits pourtant sujette à controverses, la « culture de la vitesse », « l'obsolescence programmée », la « domestication », la « dépendance » (p. 25). Pascal Minotte et Omar Rosas constatent que malheureusement les évolutions techniques sont plus rapides que les apprentissages qui en découlent. Même si l'internet nous rend tous égaux face à l'accès à l'information et à la publication, cela remet en cause les savoirs chers à l'école. Le fossé générationnel reste nécessaire à la construction d'une identité personnelle tout en portant « un regard bienveillant et confiant » (p. 33). Les avis nuancés dans ce chapitre nous amènent à réfléchir sans prendre parti. Ce souci sera exprimé tout au long de l'ouvrage.

Dans le chapitre 2, Annabelle Klein (pp. 37-65) se penche sur la construction de l'identité rendue complexe par le numérique et les médias actuels. Elle énonce clairement la continuité avec le chapitre précédent pour conforter cette unité. La notion de traçabilité est évoquée ainsi que l'intimité devenue « extimité » avec internet. Elle étudie pour cela brièvement l'évolution des « dispositifs d'autopublication » (p. 4l). Parmi eux, premières apparues, les pages personnelles ou homepage, des « lieux de soi » (p. 43) rendant les frontières sphères publiques et privées poreuses. Elle met ainsi les deux terrains en tension: «Toute page personnelle est publique » (p. 45). Cette porosité est aussi liée au jeu des métaphores attachées à l'habitat. Ensuite, avec le blog cet aspect s'est transformé pour devenir une invitation à la co-construction, une activité sociale (p. 49). Enfin apparaissent les réseaux sociaux imposant une nouvelle dualité entre méfiance et confiance.Avec Facebook, c'est le partage qui compte, le souci des risques encourus reste secondaire. Malgré la prise de conscience de ces phénomènes d'exposition, les pratiques ne se modifient pas.

Au chapitre 3 (pp. 67-94), Pascal Minotte et Arnaurd Zarbo établissent le développement de la sphère numérique et la place tenue par les écrans aux différents stades de la vie du jeune. L'intérêt porté aux écrans à n'importe quel âge inquiète parents et enseignants. Ces deux spécialistes ne remettent absolument pas en cause les outils proposés qui peuvent avoir des côtés intéressants dans certains apprentissages, mais ils mettent en garde surtout sur les risques d'exposition prolongée avec beaucoup de bienveillance, « les parents font ce qu'ils peuvent » (p. 73). Le bébé est trop rapidement confronté à cet environnement dans la sphère familiale entre passivité télévisuelle et interactivité de la tablette, des objets qui ne doivent pas prendre le pas sur les objets physiques. La scolarisation est envisagée ensuite comme une évolution des intérêts avec l'apprentissage de la lecture et une « capacité de décentration » (p. 78), écran et vie quotidienne se séparent, réalité et fiction aussi. Malheureusement, l'internet peut proposer des contenus inadaptés et l'accompagnement parental encore possible est à favoriser plus que des filtres automatiques. L'adolescent, lui, recherche l'autonomie et l'entrée « dans la norme » (p. 80) par la musique, puis par les réseaux sociaux. Ceci inquiète des parents qui reconnaissent le manque de discernement dans 
les conséquences de ses actes. Faire ses propres choix, construire son identité à travers ce désir de communiquer et d'appartenir à un groupe social, sont essentiels dans le « processus d'individuation 》 (p. 9l). Les auteurs insistent sur le fait de ne pas forcément s'alarmer, sauf pour des jeunes déjà fragiles psychologiquement.

Le chapitre 4 (pp. 95-|3|) évoque la fragilité de nos jeunes face aux images à caractère sexuel ou pornographique dont la diffusion a augmenté depuis l'apparition de l'internet. Arnaud Zarbo, psychologue, reprend ici les notions d'intimité et d'extimité, au centre de l'ouvrage. II plonge au cœur de la problématique de l'adolescence et ses enjeux. Cette étape est bouleversante et déstabilisante pour l'adolescent. L'image des autres est importante mais surtout l'image de soi, celle qu'il renvoie aux autres. Elle peut aussi s'avérer dangereuse lorsqu'elle donne lieu à du harcèlement. Le recours à l'internet pour en savoir plus sur la sexualité reste simple, anonyme, une source d'informations inépuisable, mais aussi un lieu d'échange. Discuter de ces choses-là en famille reste encore un sujet tabou. Aussi l'auteur suggère-t-il de s'ouvrir aux questions de nos jeunes même s'il reconnait les difficultés de cette situation : parler des risques et, surtout, faire émerger chez eux une pensée critique.

Le chapitre 5 (pp. 133-164) s'inscrit dans une approche psychodynamique des adolescents et des réseaux sociaux, rechercher le plaisir en évitant tout déplaisir. L'adolescence est une période transitoire marquée par de profondes modifications à la fois physiques et psychologiques enfouies dans l'inconscient. L'apparition du web a modifié les façons de penser des jeunes, il n'a plus les mêmes intérêts qu'avaient ses parents au même âge. Yann Leroux propose ici une petite histoire de l'internet jusqu'aux réseaux sociaux. Chez l'adolescent, le réseau social a un intérêt surtout dans le lien qui unit les profils entre eux (p. 139), les intérêts communs. II en conclut que le SMS est toujours privilégié parce que plus direct. S'appuyant encore sur des dualités, présence/absence, enthousiasme/ inquiétude, le contact reste constant grâce au smartphone. Il est question également des « pulsions voyeuristes » révélées par les réseaux sociaux (p. I50) au travers des désirs satisfaits : agressifs, érotiques ou narcissiques. L'adolescent peut raconter sa vie privée, tout en voilant sa personnalité. Mais cette façade publique du numérique peut devenir dangereuse. La notion de sérendipité, une expérience du hasard sur laquelle se fondent aussi les réseaux sociaux est évoquée. L'attrait le plus intéressant pour l'adolescent reste la possibilité de former des communautés, des groupes qui lui ressemblent. Le réseau social est un nouveau « tiers lieu », un espace d'interaction dont il faut aussi toujours se méfier : la vigilance est de mise.

Le dernier chapitre (pp. 165-188) expose les comportements excessifs face aux jeux vidéo et à internet. Les écrans nous envahissent et nous nous y connectons dès que possible. Cette question sociétale nous concerne donc tous et à tous les niveaux de l'activité humaine, travail et temps libre (p. 166). Pascal Minotte et Omar Rosas soulignent ici le caractère chronophage entré en concurrence avec d'autres activités. Il est nécessaire de distinguer l'outil et l'usage qui en est fait. Les angoisses se sont déplacées des écrans aux nouvelles technologies passant de la passivité télévisuelle à l'interactivité de l'internet ou du jeu vidéo. Pour aider le jeune compulsif, les parents doivent adopter une attitude compréhensive, partager son expérience, communiquer sur sa passion au lieu de le censurer ou de pratiquer l'évitement. L'usage des jeux vidéo n'est pas forcément problématique, sauf quand il envahit la vie quotidienne même hors du jeu, empêchant la concentration, le sommeil. Ces situations proviennent souvent d'une autre pathologie : dépression ou anxiété. Le jeune se réfugie dans le jeu, une réponse à certains de ses besoins. Les auteurs proposent aussi un inventaire de questions à poser au jeune compulsif pour aider les « incultes » en matière de jeux vidéo (p. 184). Du côté des réseaux sociaux et de l'internet, leur usage compulsif n'est pour l'instant pas vraiment ressenti comme une véritable addiction.

L'ouvrage se termine sur un résumé des différents chapitres. Retenons que la révolution numérique est marquée par l'opposition entre avantages et risques: la solution n'est pas de prendre parti mais plutôt la « quête de perspectives critiques 》 (p. 189). Pour agir au mieux, nous devons engager une compréhension de l'expérience des jeunes à travers les nouvelles technologies. Un problème perdure, celui des traces laissées. L'extimité n'existe que lorsque le besoin d'intimité est satisfait et les réseaux sociaux tels que Facebook n'y aident pas, les traces étant toujours difficilement effaçables. La thématique sur la vie sexuelle et affective des adolescents à l'ère numérique reste la plus anxiogène pour les parents.

La structure globale du livre conduit le lecteur dans la psychologie comportementale des adolescents en proie aux plus grands bouleversements de leur vie. Malgré tout, cet ouvrage reste un peu trop théorique si l'on cherche des réponses concrètes. Les conseils sont très, voire trop, techniques pour le public ciblé, même si beaucoup de notions importantes sont reprises et expliquées. 
Pour autant tout, il répond à certaines des questions des parents sur les problèmes comportementaux des adolescents en donnant quelques clés.

Florence Michet

Mica, université Bordeaux-Montaigne, F-33600 florence.michet@etu.u-bordeaux-montaigne.fr

\section{Michel MARCOCCIA, Analyser la communication numérique écrite}

Paris,A. Colin, coll. Icom. Série Discours et communication, 2016, 160 pages

Voici un livre très utile pour tous les linguistes qui se posent des questions sur les nouvelles formes de communication numérique. Un livre clair, bien organisé, avec des explications et des descriptions précises de ce qu'est la « communication numérique écrite » (ou « communication écrite en ligne ») - un hyper-genre sous-tendant des formes émergeant actuellement dans notre environnement verbal sur l'internet. Le volume est destiné aux étudiants en sciences de l'information et de la communication, en sciences politiques et en sciences du langage, et parait dans la collection Icom dirigée par Dominique Maingueneau.

On doit ce livre à un spécialiste du domaine, Michel Marcoccia, qui a déjà apporté beaucoup de contributions sur divers discours numériques : forums de discussion entre adolescents, courrier des lecteurs, forums des partis politiques et des entreprises, polylogues en ligne, etc. Ce chercheur définit cette forme de communication dès son introduction (pp. 7-I2) : «Cette dénomination renvoie aux nombreuses productions écrites réalisées par le moyen de l'internet ou de la téléphonie mobile » et contient « le courrier électronique, le forum de discussion, la liste de diffusion, le tchat, la messagerie instantanée, le SMS (ou texto), le blog, les plateformes de réseaux sociaux » (p.7). Des formes de la communication verbale qui interpellent fortement la linguistique générale et la linguistique du discours.

Le livre s'organise en trois parties, destinées à décrire les dispositifs utilisés, les spécificités des écrits numériques (essentiellement conversationnels et « contextualisés», p. II) et, enfin, quelques particularités pragmatiques (structuration et formats de participation, la construction des identités numériques et des relations interpersonnelles, etc.).

Chacune de ces parties est sous-divisée en chapitres, dans une démarche très systématique pour le lecteur, où sont également intégrés les apports des nombreux chercheurs ayant contribué à la description du domaine. Un sous-chapitre dresse un bref historique des dispositifs CMO (communication médiée par ordinateur) et CMT (communication médiée par téléphone) (pp. 17-21), passant en revue les technologies Minitel et les formes asynchrones et synchrones de communication (formes du courrier électronique, forums de discussion et listes de diffusion, pour les premières ; tchat et messagerie instantanée grand public, pour les secondes), pour arriver aux blogs, aux réseaux sociaux Facebook, Linkedln ou aux plateformes de wikis.

Un autre sous-chapitre explicite la profusion de termes utilisés pour dénommer ce type de communication non encore « stabilisé » dans l'esprit des utilisateurs ou dans la terminologie des chercheurs. Ainsi « électronique » et « numérique » se font-ils concurrence, en anglais ou en français, avec des dénominations comme: electronic communication, discours électronique médié, communication électronique scripturale, écriture numérique, écrits d'écrans, discours d'internet, activités technoscripturales, computer conversation, written interactive discours, écrit interactif et dialogique etc. (pp. 21-23), catégories quasi-synonymes. $\mathrm{Ce}$ côté terminologique s'avère intéressant pour le chercheur qui devra dorénavant s'habituer à utiliser de nouvelles catégories comme : persistance des messages (pp. 26-30), culture de « lulz » (Rire aux dépens d'autrui) (p. 33), flames (injures) (p. I34) pseudonymat, trolles (internaute postant sur des espaces de discussion des messages provocateurs) (p. 137), genre participatif (p. 139), identité numérique (p. 145), autonymes et avatars (p. 149) - toutes définies dans ce livre dès leurs apparitions dans le texte ; tout comme des abréviations nouvelles, telles CMDA (Computer-Mediated Discourse Analysis, p. 28), ASV (Âge, sexe, ville), pour la description de soi sur internet (p. 149). Nous ne mentionnons pas ici les abréviations déjà connues pour tout utilisateur d'internet et de messages électroniques.

Michel Marcoccia met en avant les avantages que peuvent tirer les chercheurs en linguistique de ces écrits sur internet, dont la taille (c'est un immense « corpus »!) et l'accessibilité permettent des reprises et des recherches beaucoup plus amples, une meilleure représentativité, sans parler du fait que la plupart s'offrent comme déjà transcrits par leurs producteurs (pp. 38, 39). Un problème qui reste pourtant non résolu est celui de la déontologie de ces reprises, et ce, de par la difficulté d'identifier si certains terrains sont publics ou privés (pp. 42-47). 